\title{
Research Paper: The Association Between Hand Grip Strength and Hand Span of Dominant and Non-dominant Hand of Undergraduate Physiotherapy Students
}

\author{
Sajida Bibi Noonari ${ }^{1}$, Bakhtawar Samejo ${ }^{2^{*}}$, Muhammad Haris Nonari ${ }^{3}$ Q \\ 1. Department of Physiotherapy, National Institute of Physiotherapy and Rehabilitation Sciences, Liaquat University of Medical and Health Sciences, \\ Pakistan. \\ 2. Department of Physiotherapy, Bhitai institute of Physiotherapy and Rehabilitation Sciences, Riphah International University, Pakistan \\ 3. Orthosis Prosthesis Department, Liaquat University of Medical and Health Sciences, Pakistan.
}

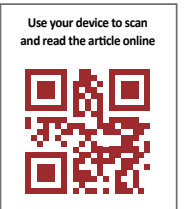

Crtation: Bibi Noonari S, Samejo B, Haris Nonari M. The Association Between Hand Grip Strength and Hand Span of Dominant and Non-dominant Hand of Undergraduate Physiotherapy Students. Journal of Modern Rehabilitation. 2019; 13(4):193198. http://dx.doi.org/10.32598/JMR.13.4.193

http://dx.doi.org/10.32598/JMR.13.4.193

Article info:

Received: 13 Apr 2019

Accepted: 23 Jul 2019

Available Online: 01 Oct 2019

Keywords:

Handgrip strength, Handspan,

Dominant hand strength,

Handgrip and span ratio

\begin{abstract}
Introduction: Health-related physical fitness requires muscular strength to perform physical activities. The handgrip test is used to assess the muscular strength of the hand. The flexor muscle strength in palmar, thenar, and hypothenar areas of the hand collectively form the Handgrip Strength (HGS). However, the extensor mechanism, including the intrinsic musculature, plays a minor role in producing handgrip strength. Also, each finger plays an essential role in handgrip strength. This study aims to identify the association between the handgrip strength and the handspan of the dominant and non-dominant hands of undergraduate physical therapy students.
\end{abstract}

Materials and Methods: A cross-sectional observational study was conducted on undergraduate physical therapy students. Different public and private physical therapy schools and universities in Karachi City, Pakistan were chosen to collect the data with an estimated sample size of 100 undergraduate physical therapy students. The sampling was done by the non-probability convenient sampling method. The inclusion criteria were undergraduate physiotherapy students (from both genders) and willing to participate in the study.

Results: The Pearson test was used to determine the significant correlation between handgrip strength and handspan. The results showed the mean and standard deviation of handgrip strength (dominant hand) was $25.96 \pm 11.538$, of handgrip strength in $\mathrm{Kg}$ (non-dominant hand) was $24.93 \pm 12.336$, and of handspan length in $\mathrm{Cm}$ of the dominant hand was 20.2910 \pm 1.78671 . The present study showed a positive association between handgrip strength and handspan. The handgrip strength of the non-dominant hand was higher than the dominant handgrip strength values. Handspan was also higher in the non-dominant hand compared with the dominant hand, but this difference is not significant.

Conclusion: The handgrip strength of physical therapy students had a positive association with the handspan. The handspan and dominant hand influence handgrip strength.

* Corresponding Author: 


\section{Introduction}

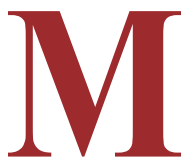

uscular strength is one of the components of health-related physical fitness and refers to the ability to perform activities that need muscular force. Handgrip is one of the standard tests to assess muscular strength of the hand [1]. Handgrip Strength (HGS) is a summation of the power of the flexor muscles of the palmar, thenar, and hypothenar areas of the hand. The extensor mechanism, including the intrinsic musculature, plays a minor role in producing handgrip strength. However, each finger plays an essential role in handgrip strength. Several research studies were conducted to identify the contribution of each finger on handgrip strength $[2,3]$. Muscles hypertrophy, range of motion, gender, and types of muscle fiber, muscles coordination, and muscles shortening velocity are the internal factors that affect muscular strength. Besides, internal factors, some external factors like motivation, type of training, time of day, and anabolic steroids also affect muscular strength [4]. Handgrip strength is a predictor of several diseases, such as heart, cerebrovascular, cognitive disorders, and a higher risk of fractures. Several studies have supported the correlation of handgrip with nutritional status, bone mineral content, hand length, body mass index, age, and upper arm circumference [5]

Handgrip strength declines after midlife [5]. Handgrip strength is usually measured with a handgrip dynamometer. It gives reliable and useful information about overall health [1]. The handgrip strength test is a simple and economical method to assess handgrip strength. The test results also provide practical information about muscles, bone, nerve, and joint disorders [6]. A weak handgrip is a predictor of poor clinical outcomes; even a weak handgrip with aging predicts functional limitation, disability, and other health-related complications like cardiovascular diseases [7]. Handgrip assessment helps evaluate the patient's weakness and also his or her progress during the rehabilitation phase and the effectiveness of the treatment [5].

Generally, handgrip strength is done on the dominant hand to identify the highest strength value. Studies support $10 \%$ higher handgrip strength of the dominant hand as compared to non-dominant hand [8]. Hand length, hand shape, and body height ratio determine grasping capability while palmar length and width ratio, and other anthropometric parameters determine grip strength [9]. The effects of hand dimensions like finger span, finger lengths, perimeters of the hand, and hand shape were also studied in different populations [10]. One study was conducted to evaluate HGS and handspan among healthy adults [5].

Another study was conducted to identify the association between HGS and anthropometric measurements. Results found that dominant and non-dominant handgrip is positively associated with height, weight, hand span, wrist circumstance. Also, hand length is associated with a handgrip, while body mass index and handspan have no significant association with handgrip in healthy Indian females [11].

A study was conducted to investigate the handgrip strength and grip control strength as a predictor of upper extremity function. The study concluded that HGS was influenced by hand size and grip span in both hands and for optimal grip span dynamometer should be adjusted when performing the HGS assessment test [8]. Physiotherapists should have good grip strength for mobilization, manipulation, transferring, and ambulation. The nursing profession needed a firm hand grip to move or lift or inject drugs to the patient. The importance of handgrip strength varies in the different health care professions. However, it is an essential factor for planning and evaluating rehabilitation outcomes [12].

There is no evidence of published scientific articles on the association between hand span and handgrip strength among physical therapy students in Karachi, Sindh. The study aims to identify the association between handgrip strength and a hand span of dominant and non-dominant hand among undergraduate physical therapy students.

\section{Methods}

A cross-sectional observational study was conducted on undergraduate physical therapy students. The study data were collected from different public and private physical therapy schools and universities in Karachi, with an estimated sample size of 100 undergraduate physical therapy students. The sampling was done by a non-probability convenient sampling method. The participants were selected with the following inclusion criteria: undergraduate physical therapy students, both genders (male and female), willingness to participate in the study. Participants would be excluded from the study if they were unable to give consent or have any cognitive deficits, those with a recent hand injury or fracture, subjects with hand deformity, and those who were unwilling to participate. 


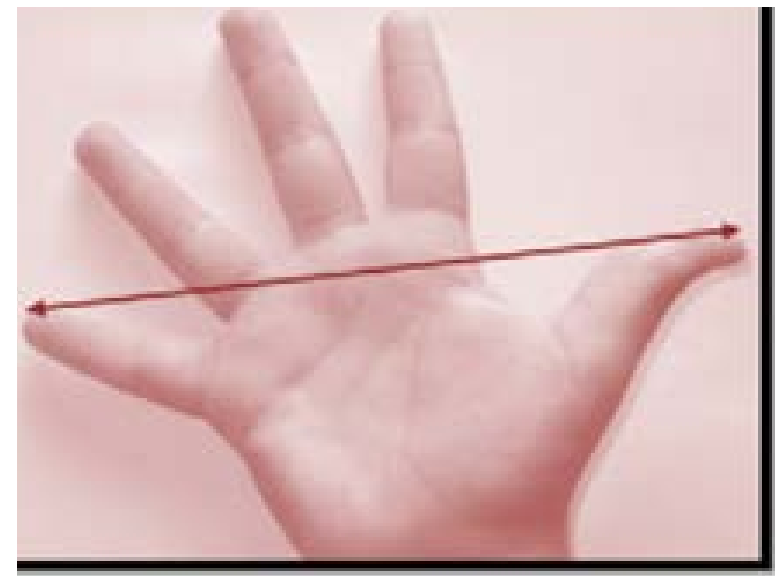

$J \mathrm{MR}$

Figure 1. Measurement of handspan of a participant

\section{Study Procedure}

Each participant signed written informed consent before data collection. Dynamometer and measuring scale were used as an assessment tool.

\section{Handspan}

Handspan was measured in $\mathrm{Cm}$ on the dominant hand of the participants from the tip of the thumb to the tip of the small finger with the hand opened as wide as possible.

\section{Handgrip Strength}

The maximum handgrip strength was measured with a handgrip dynamometer. During taking measurements, participants maintained the standard bipedal position during the entire test with the arm and upper arm at a $90^{\circ} \mathrm{C}$. Each participant performed the test three times approximately for $3 \mathrm{~s}$, then relaxed, and next does the same procedure for three more times. Participants took a 10 -s break between each repetition the reading was noted in $\mathrm{Kg}$.

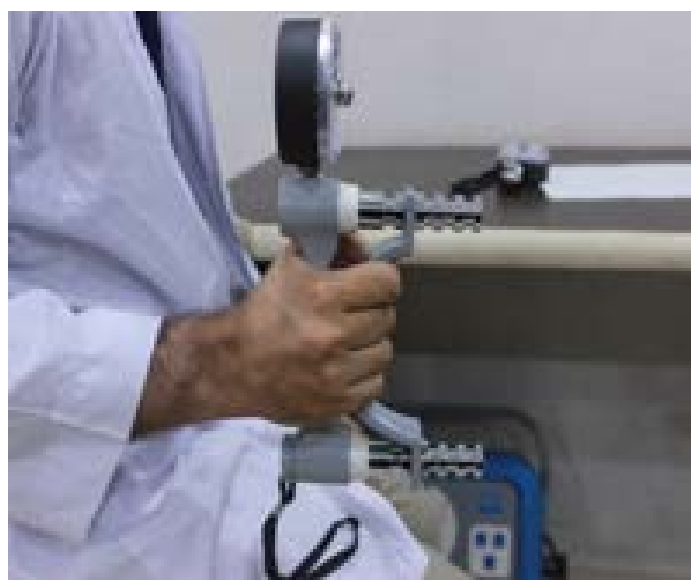

$J M R$

Figure 2. Measurement of handgrip strength of a participant via dynamometer

\section{Results}

The collected data were analyzed in SPSS 23.0. Descriptive analyses were also calculated. The Pearson test was used to determine the significant correlation between handgrip strength and handspan.

Table 1 shows the mean and standard deviation of handgrip strength in $\mathrm{Kg}$ (dominant hand) is $25.96 \pm 11.538$, handgrip strength in $\mathrm{Kg}$ (non-dominant hand) is $24.93 \pm 12.336$, handspan of dominant hand in Cm 20.2910 \pm 1.78671 .

Table 2 shows the results of the Pearson correlation of handgrip and handspan; the value of the Pearson handgrip (dominant hand) and handgrip (non-dominant hand) is 0.940 with the 0.000 level of significance (correlation is significant at the level of 0.01 level). Also, the value of the Pearson handgrip (non-dominant hand) and handspan are 0.714 with 0.000 level of significance (correlation is significant at the level of 0.01 level).

\section{Discussion}

The preliminary aim of this study is to identify the association between handgrip strength and the handspan

Table 1. Mean $\pm S D$ of Handgrip and Handspan of dominant and non-dominant hand measurements

\begin{tabular}{ccccc}
\hline Variables & N & Min. & Max. & Mean \pm SD \\
\hline Handgrip strength in Kg (dominant hand) & 100 & 10 & 55 & $25.96 \pm 11.538$ \\
Handgrip strength in Kg (non-dominant hand) & 100 & 6 & 55 & $24.93 \pm 12.336$ \\
Handspan in Cm (dominant hand) & 100 & 17.00 & 24.00 & $20.2910 \pm 1.78671$
\end{tabular}


Table 2. The results of the Pearson correlation of handgrip strength and handspan

\begin{tabular}{|c|c|c|c|c|}
\hline Variables & Pearson Correlation & $\begin{array}{l}\text { Handgrip Strength } \\
\text { (Dominant Hand) }\end{array}$ & $\begin{array}{l}\text { Handgrip Strength } \\
\text { (Non-Dominant } \\
\text { hand) }\end{array}$ & $\begin{array}{c}\text { Handspan (Domi- } \\
\text { nant Hand) }\end{array}$ \\
\hline $\begin{array}{l}\text { Handgrip strength in } \mathrm{Kg} \\
\text { (Dominant hand) }\end{array}$ & $\begin{array}{c}\text { The Pearson correlation } \\
\text { Sig. level } \\
\text { N }\end{array}$ & 1 & $\begin{array}{c}0.940 \\
0.000 \\
100\end{array}$ & $\begin{array}{c}0.710 \\
0.000 \\
100\end{array}$ \\
\hline $\begin{array}{l}\text { Handgrip strength in Kg } \\
\text { (Non-dominant hand) }\end{array}$ & $\begin{array}{c}\text { The Pearson correlation } \\
\text { Sig. level } \\
\text { N }\end{array}$ & $\begin{array}{c}0.940 \\
0.000 \\
100\end{array}$ & 1 & $\begin{array}{c}0.714 \\
0.000 \\
100\end{array}$ \\
\hline $\begin{array}{l}\text { Handspan in } \mathrm{Cm} \text { (Domi- } \\
\text { nant hand) }\end{array}$ & $\begin{array}{c}\text { Pearson correlation } \\
\text { Sig. level } \\
\mathrm{N}\end{array}$ & $\begin{array}{c}0.710 \\
0.000 \\
100\end{array}$ & $\begin{array}{c}0.714 \\
0.000 \\
100\end{array}$ & 1 \\
\hline
\end{tabular}

of the dominant and non-dominant hand of the undergraduate physical therapy students. The participants were selected via non-probability convenient sampling techniques. The present study showed a positive association between Handgrip Strength (HGS) and handspan. The handgrip strength of the non-dominant hand was higher than the right handgrip strength values. Handspan was also higher in the non-dominant hand compared to the dominant hand, but it is not significant [13]

In another study, a positive correlation was found between handgrip strength and handspan among healthy adults. Furthermore, a significant difference was found between handgrip and handspan, in different age groups [5]. Moreover, the study found a positive correlation between handgrip strength and hand span.

A study was conducted to evaluate HGS and handspan among healthy adults. The results revealed that the hand span is positively associated with handgrip strength [5]. One study was conducted to evaluate the handgrip strength and grip control strength as the predictors of upper extremity function. The findings revealed that hand size is positively associated with the highest handgrip strength in both hands but significantly affect grip control strength. The study concluded that HGS was influenced by hand size and grip span in both hands, and for optimal grip span, the dynamometer should be adjusted when performing the HGS assessment test [6].

\section{Conclusion}

The handgrip strength of physical therapy students had a positive association with their handspan. The handgrip strength should be improved among physical therapist students because, in the future, these physical therapists need hand strength for proper force implications for the manual therapy and management of their patients. Fur- ther studies need to be conducted to identify the association of handgrip strength with other variables.

\section{Ethical Considerations}

\section{Compliance with ethical guidelines}

All ethical considerations were maintained during collection of data, written informed consent were taken prior to participation

Funding

This research did not receive any specific grant from funding agencies in the public, commercial, or not-forprofit sectors.

\section{Authors contributions}

Conceptualization, funding, writing original draft, review and editing: all authors; methodology: Bakhtawar samejo; Resources: Bakhtawar samejo; Supervision: Fahad Farooq lasi.

\section{Conflict of interest}

The authors declared no conflict of interest.

\section{References}

[1] Matute-Llorente Á, González-Agüero A, Vicente-Rodríguez G, Casajús JA. Hand span influences optimal grip span in adolescents with Down syndrome. Nutrición Hospitalaria. 2017; 34(3):626-31. https://www.researchgate.net/publication/317814894

[2] Liao WC, Wang CH, Yu SY, Chen LY, Wang CY. Grip strength measurement in older adults in taiwan: A comparison of three 
testing positions. Australasian Journal on Ageing. 2014;33(4):27882. [DOI:10.1111/ajag.12084] [PMID]

[3] Cha SM, Shin HD, Kim KC, Park JW. Comparison of grip strength among 6 grip methods. The Journal of Hand Surgery. 2014; 39(11):2277-84. [DOI:10.1016/j.jhsa.2014.06.121] [PMID]

[4] de Andrade Fernandes A, Natali AJ, Vieira BC, do Valle MAAN, Moreira DG, Massy-Westropp N, et al. The relationship between hand grip strength and anthropometric parameters in men. Archivos de Medicina del Deporte. 2014; 31(3):160-4. https:/ / pdfs. semanticscholar.org/32dc/53916163934d6b459d39e7e583a1b32 42c68.pdf

[5] Alahmari KA, Silvian SP, Reddy RS, Kakaraparthi VN, Ahmad I, Alam MM. Hand grip strength determination for healthy males in Saudi Arabia: A study of the relationship with age, body mass index, hand length and forearm circumference using a hand-held dynamometer. Journal of International Medical Research. 2017; 45(2):540-8. [DOI:10.1177/0300060516688976] [PMID] [PMCID]

[6] Liao KH. Hand grip strength in low, medium, and high body mass index males and females. Middle East Journal of Rehabilitation and Health. 2016; 3(1):e33860. [DOI:10.17795/mejrh-33860]

[7] García-Hermoso A, Carrillo HA, González-Ruíz K, Vivas A, Triana-Reina HR, Martínez-Torres J, et al. Fatness mediates the influence of muscular fitness on metabolic syndrome in Colombian collegiate students. PLoS One. 2017; 12(3):e0173932. [DOI:10.1371/journal.pone.0173932] [PMID] [PMCID]

[8] Klippel JH, Stone JH, White PH. Primer on the rheumatic diseases. Berlin: Springer Science \& Business Media; 2008.

[9] Abe T, Loenneke JP. Handgrip strength dominance is associated with difference in forearm muscle size. Journal of Physical Therapy Science. 2015; 27(7):2147-9. [DOI:10.1589/jpts.27.2147] [PMID] [PMCID]

[10] Hemberal M, Doreswamy V, Rajkumar S. Study of correlation between hand circumference and Maximum Grip Strength (MGS). National Journal of Physiology, Pharmacy and Pharmacology. 2014; 4(3):195-7. [DOI:10.5455/njppp.2014.4.280220142]

[11] Shah UN, Sirajudeen MS, Somasekaran PK, Mohasin N, Shantaram $\mathrm{M}$. The association between hand grip strength and hand dimensions in healthy Indian females. International Journal of Current Research and Review. 2012; 4(2):36-42. http:/ /ijcrr.com/ article_html.php?did=1963

[12] Zakariya M, Shah UN, Mohan N, Saad Sh, Shaikhji NM. Analysis of power grip and pinch grip among health care professionals. International Journal of Physiotherapy. 2016; 3(1):45-52. [DOI:10.15621/ijphy/2016/v3i1/88909]

[13] Chahal A, Kumar B. Relationship of hand anthropometry and hand grip strength in junior basketball boys. International Journal of Health Sciences \& Research. 2014; 4(11):166-73. https:// www.semanticscholar.org/paper/Relationship-of-Hand-Anthropometry-and-Hand-Grip-in-Chahal-Kumar/2018997b29217 9309f $2575 \mathrm{ac} 13 \mathrm{~b} 672 \mathrm{c} 75 \mathrm{db} 2418 \mathrm{c}$ 
This Page Intentionally Left Blank 